\title{
Schlaf - nächtlicher Verkehrslärm - Streß - Gesundheit: Grundlagen und aktuelle Forschungsergebnisse
}

\author{
Teil I: Grundlagen
}

\author{
Von C. Maschke, H. Ising und K. Hecht
}

\section{Einleitung}

Zwei gesundheitsrelevanten Erscheinungen und ihrer Interaktion wird gegenwärtig nicht die gebührende gesellschaftliche Bedeutung beigemessen:

(1) dem Schlaf,

(2) dem Lärm und

(3) der Beziehung zwischen Lärm und Schlaf.

Auf die gesundheitlichen Auswirkungen des gestörten Schlafes wird $1995 \mathrm{im}$ Weißbuch "Schlafmedizin « [1] mit Nachdruck verwiesen. 20 Millionen Deursche leiden an Einschlaf- und Durchschlafstörungen. Davon sind 8,5 Millionen so stark erkrankt, daß sie behandlungsbedürftig sind. 3,3 Millionen Menschen in Deutschland leiden an ständiger Tagesmüdigkeit infolge schlechter Schlafqualität. Das hat z. B. eine Verminderung der Leistungsfähigkeit und der Verkehrssicherheit zur Folge. So sind $24 \%$ aller Verkehrstoten 1991 auf den bayerischen Autobahnen auf "Einschlafen am Steuer « zurückzuführen.

Die in Tabelle 1 angeführte Epidemiologie [2] unterstreicht die im Weißbuch »Schlafmedizin « dargelegte kritische Situation.

In der Internationalen Klassifikation der Schlafstörungen (ICSD) sind insgesamt 88 verschiedene Störungen (des Schlafes) verzeichnet [3]. Darüber hinaus verweist Moore-Ede [4] darauf, daß ein gestörter Schlaf nicht nur schlechthin eine verminderte Erholung darstellt, sondern die Zeitstruktur des Menschen zerstört, woraus sich schwerwiegende gesundheitliche Störungen und Schäden entwickeln können.

Nach der WHO-Definition haben chronische Schlafstörungen Krankheitswert, denn sie beeinträchtigen:

- Leistungsfähigkeit,

- psychisches Wohlbefinden,

- kindliche Kondition,

- das Immunsystem,
- soziale Kommunikation,

- Verkehrstüchtigkeit u.v.m.

Sie sind Risikofaktoren für

- psychosomatische Erkrankungen, insbesondere Herzkreislaufkrankheiten,

- Neurosen,

- Angst,

- Agressivität.

Ein ungestörter Schlaf bewirkt Gesundheit, Wohlbefinden, Leistungsfähigkeit, Optimismus, Erfolge. Das Maß für einen guten erholsamen Schlaf ist die Ausgewogenheit der Regulation der drei Kardinalzustände des Menschen $[10,11]$

- Wachsein,

- Schlaf (Non-REM-Schlaf),

- Traumschlaf (REM-Schlaf).

Sobald ein Ungleichgewicht in diesem System auftritt, können die angeführten
Gesundheitsstörungen auftreten. Der Schlaf ist als eine periodisch ablaufende psychobiologische Funktion zu betrachten [12]. Das in Abbildung 1 dargestellte Schema verdeutlicht die Zusammenhänge zwischen den psychischen und biologischen Komponenten des Schlafs. Das Schlaferleben ist an den Non-REM-Schlaf, das Traumerleben an den REM-Schlaf gebunden.

Bei einer Untersuchung des Schlafs sollte stets ein psychisches und ein biologisches Instrumentarium an Methoden verwendet werden, wie es $z$. B. in den Studien von Maschke et al. $[13,14]$ erfolgte.

Die biologische Komponente wird in der schlafmedizinischen Diagnostik mittels der elektrophysiologischen Schlafpolygraphie (EEG, EMG, EOG, EKG, Atmung u. a.) untersucht und als Schlafzyklogramm (Schlafprofil) darge-

Tabelle 1: Prävalenz verschiedener Schlafstörungen in Deutschland und Europa (ausgenommen Rußland)

\begin{tabular}{|c|c|c|c|}
\hline Erkrankung & Prävalenz & $\begin{array}{l}\text { Anzahl der } \\
\text { Patienten in } \\
\text { Deutschland } \\
\text { (Bevölkerung } \\
80 \text { Mio.) }\end{array}$ & $\begin{array}{l}\text { Anzahl der } \\
\text { Patienten in } \\
\text { Europa (außer } \\
\text { Rußland) } \\
\text { (Bevölkerung } \\
\text { 550 Mio.) }\end{array}$ \\
\hline $\begin{array}{l}\text { Leichte Ein-und } \\
\text { Durchschlafstörungen }\end{array}$ & $\begin{array}{l}23-51 \% \\
{[5]}\end{array}$ & 18,4 - 40,8 Mio. & 126,5 - 280,5 Mio. \\
\hline $\begin{array}{l}\text { Schwere Ein- und } \\
\text { Durchschlafstörungen }\end{array}$ & $10-26 \%$ & $8-20,8$ Mio. & 55-143 Mio. \\
\hline Narkolepsie & $\begin{array}{l}0,065 \% \\
{[6]}\end{array}$ & 52000 & 357500 \\
\hline $\begin{array}{l}\text { Idiopathische } \\
\text { Hypersomnie }\end{array}$ & $\begin{array}{l}0,0074 \% \\
{[7]}\end{array}$ & 5920 & 40700 \\
\hline $\begin{array}{l}\text { Obstruktive } \\
\text { Schlaf-Apnoe }\end{array}$ & $\begin{array}{l}1-2 \% \\
\text { (versch. Übersichten) }\end{array}$ & $800000-2$ Mio. & 5,5-11 Mio. \\
\hline $\begin{array}{l}\text { Restless-legs- } \\
\text { Syndrom }\end{array}$ & $\begin{array}{l}5 \% \\
{[8]}\end{array}$ & 4 Mio. & 27,5 Mio. \\
\hline $\begin{array}{l}\text { Periodische Bewegun- } \\
\text { gen der Unterschenkel } \\
\text { im Schlaf }\end{array}$ & $\begin{array}{l}29 \% \text { der über 50jährigen } \\
{[9]}\end{array}$ & 23,2 Mio. & 159,5 Mio. \\
\hline
\end{tabular}




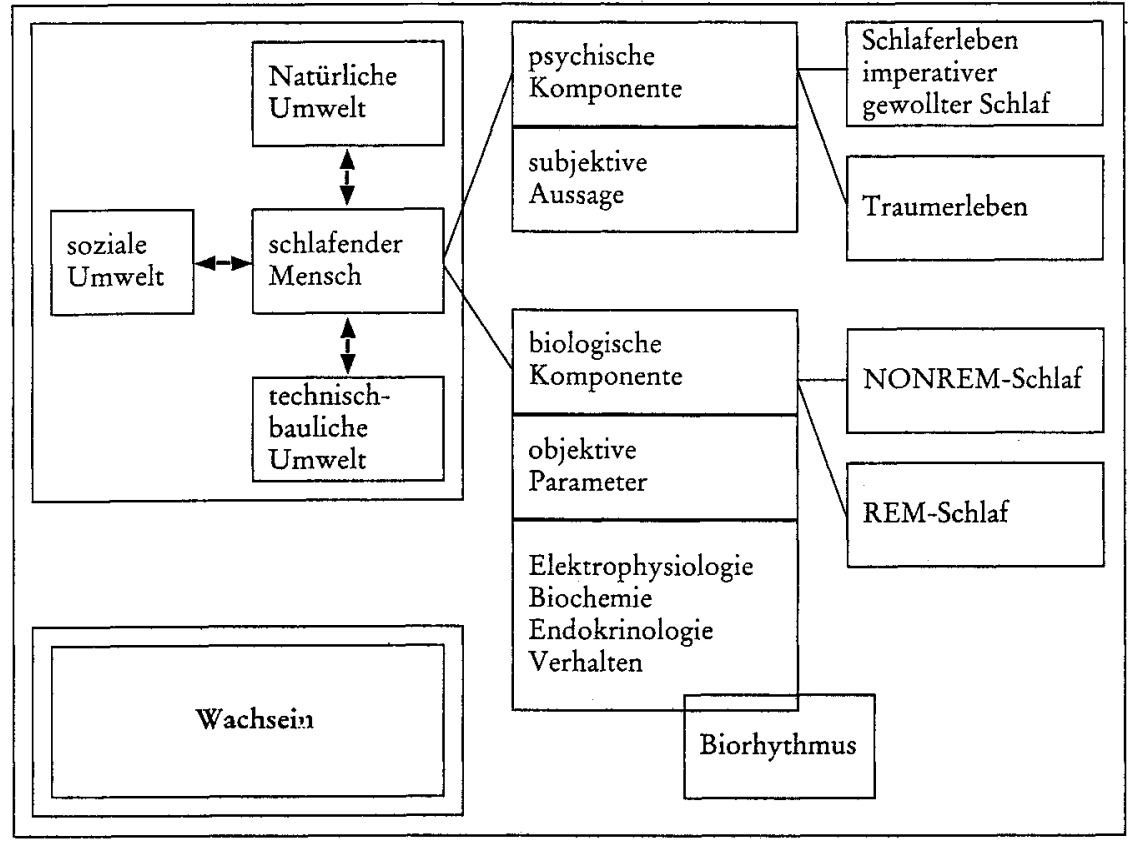

Abbildung 1: Psychische und biologische Komponenten des Schlafs [12].

stellt. Das Schlafzyklogramm enthält fünf Schlafstadien, die in ihrer zeitlichen Abfolge über der Schlafzeit aufgetragen werden. Zusätzlich werden die Zeiten erhöhter Muskelaktivität (Movementtime) und die Wachphasen eingezeichnet. Der Schlaf beginnt mit dem Übergang vom Wachzustand in das Schlafstadium 1 (NONREM 1). Nach wenigen Minuten wird das Schlafstadium 2 (NONREM 2) erreicht. Wird der Schläfer nicht gestört, erreicht er nach einer kurzen Verweildauer im Stadium 3 (NONREM 3) den Tiefschlaf (NONREM 4). Die Weckschwelle nimmt mit jeder Schlafvertiefung zu. Die Ordinatenwerte der vier Schlafstadien spiegeln diese erschwerte "Weckbarkeit" wider. Das Ende eines Schlafzyklus bildet der (meist) als Balken auf dem Niveau des Stadiums 1 verzeichnete REM-Schlaf (Traumschlaf). Im Traumschlaf ist eine EEG-Tätigkeit zu beobachten, die dem Stadium NONREM 1 sehr ähnlich ist. Dieses Schlafstadium wird durch heftige Bewegungen der Augäpfel unter den geschlossenen Lidern (Rapid Eye Movements) gekennzeichnet und daher als REM-Schlaf bezeichnet.

Im ungestörten Schlaf folgt auf eine Schlafvertiefung eine schnelle Schlafverflachung, die mit dem REM-Stadium beendet wird. Diesen Schlafzyklus "durchwandert « der Schlafende während einer achtstündigen Nacht vierbis fünfmal. Die Verweildauer in den
Schlafstadien NONREM $3+4$ nimmt mit der Schlafdauer ab, die Verweildauer im REM-Schlaf mit der Schlafdauer zu. Der zyklische Ablauf der Schlafstadien ist Teil einer ultradianen Periodik. Ein typisches Schlafzyklogramm ist in Abbildung 2 dargestellt.

Am rechten Rand des Schlafzyklogramms sind die relativen SchlafstadienZeiten für jedes Schlafstadium vermerkt. Die (relativen) SchlafstadienSchlaf eine charakteristische Verteilung Zeiten weisen bei einem ungestörten Instituts für Technische Akustik. auf. Eine deutliche Änderung dieser Schlafstadienverteilung ist ein Zeichen für einen gestörten Schlaf.

Wahrnehmung, Empfinden und Motorik sind in den verschiedenen Stadien des Schlaf-Wach-Zyklus unterschiedlich. Eine Übersicht enthält Tabelle 2.

Für die physische Erholung ist der Tiefschlaf (Deltaschlaf) und für die psychische Erholung der REM-Schlaf verantwortlich. Neuere Theorien aus der Neurobiologie unterstützen diese Erkenntnis [15]. Dem REM-Schlaf wird eine besondere Bedeutung beigemessen, die in der "Aufbereitung und Reflexion von Gedächtnisinhalten « liegt. Diese beiden Schlafzustände in rhythmischer Ausgewogenheit ablaufend bestimmen die Schlafqualität. Sie lassen sich wie in $\mathrm{Ta}-$ belle 3 dargestellt charakterisieren.

Untersuchungen von Horne [16] zeigten, daß der Verlust (Reduzierung) des Deltaschlafes durch körperliches Ruhen kompensiert werden kann. Dagegen ist der reduzierte oder eleminierte REMSchlaf nicht zu ersetzen. Ein längerer REM-Schlaf-Entzug führt zu schweren Beeinträchtigungen der geistigen Prozesse, die bereits nach zehn bis zwölf Tagen Neurosen auslösen können.

Neben den biologischen Aspekten sind auch die Aspekte der psychischen Komponente des Schlafes zu beachten. Eine von Hecht [12] durchgeführte Befragung ergab, daß über $80 \%$ der Befragten den Schlaf mit größerer Sehnsucht erwarten als das Wachsein. Begründet wurde diese Antwort damit, daß der Schlaf Geborgenheit, Genuß, Erholung

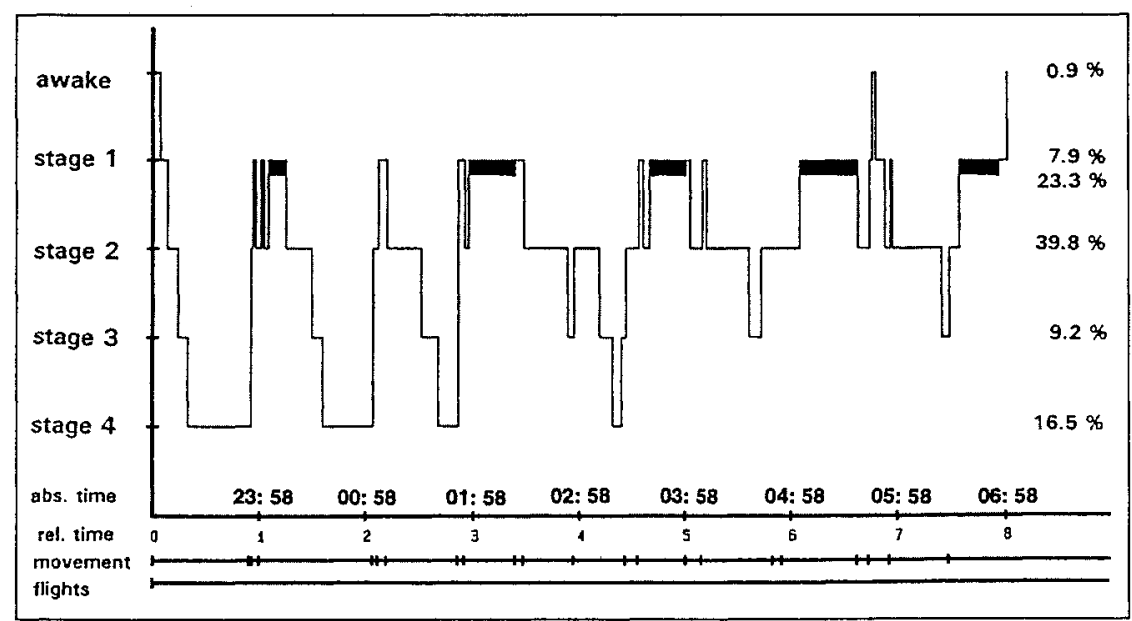

Abbildung 2: Typisches Schlafzyklogramm unter Ruhebedingungen. Die Versuchsperson schlief bei einem nächtlichen Mittelungspegel von $L_{\text {eq innen }}<30 \mathrm{~dB}(\mathrm{~A})$ im Schlaflabor des 
Tabelle 2: Wahrnehmung, Empfindung und Motorik im Schlaf-Wach-Zyklus [11]

\begin{tabular}{|c|c|c|c|c|c|c|}
\hline Indikator & $\begin{array}{l}\text { Entspanntes } \\
\text { Wachsein }\end{array}$ & NONREM I & NONREM II & NONREM III & NONREM IV & REM \\
\hline Bewußtsein & uneingeschränkt & $\begin{array}{l}\text { Aufnahme von } \\
\text { Informationen } \\
\text { noch möglich, } \\
\text { hypnagoge } \\
\text { Halluzinationen }\end{array}$ & erloschen & erloschen & erloschen & erloschen \\
\hline Weckschwelle & $\begin{array}{l}\text { Normale } \\
\text { Beantwortung } \\
\text { äußerer Reize }\end{array}$ & $\begin{array}{l}\text { Weckschwelle } \\
\text { gegenüber der } \\
\text { Umwelt erhöht }\end{array}$ & $\begin{array}{l}\text { weitere } \\
\text { Erhöhung der } \\
\text { Weckschwelle }\end{array}$ & $\begin{array}{l}\text { weiterer } \\
\text { Anstieg der } \\
\text { Weckschwelle }\end{array}$ & $\begin{array}{l}\text { Weckschwelle } \\
\text { ähnlich wie } \\
\text { Stadium III }\end{array}$ & $\begin{array}{l}\text { Weckschwelle } \\
\text { noch höher } \\
\text { im Stad. IV }\end{array}$ \\
\hline $\begin{array}{l}\text { Augen- } \\
\text { bewegungen }\end{array}$ & $\begin{array}{l}\text { schnelle und } \\
\text { langsame }\end{array}$ & langsame & keine & keine & keine & schnelle \\
\hline Motorik & $\begin{array}{l}\text { Muskeltonus } \\
\text { mittelhoch bis } \\
\text { hoch, Reflexe } \\
\text { erhalten, Bewe- } \\
\text { gungsartefakte }\end{array}$ & $\begin{array}{l}\text { Muskeltonus } \\
\text { mittelhoch bis } \\
\text { hoch, Reflexe } \\
\text { erhalten, gele- } \\
\text { gentlich Bewe- } \\
\text { gungsartefakte, } \\
\text { hypnagoge } \\
\text { Muskel- } \\
\text { zuckungen }\end{array}$ & $\begin{array}{l}\text { Muskeltonus } \\
\text { mittelhoch, } \\
\text { Reflexe er- } \\
\text { halten, gele- } \\
\text { gentlich Bewe- } \\
\text { gungsartefakte }\end{array}$ & $\begin{array}{l}\text { Muskeltonus } \\
\text { mittelhoch, } \\
\text { Reflexe er- } \\
\text { halten, keine } \\
\text { Bewegungs- } \\
\text { artefakte }\end{array}$ & $\begin{array}{l}\text { Muskeltonus } \\
\text { niedrig bis } \\
\text { mittelhoch, } \\
\text { keine Bewe- } \\
\text { wegungs- } \\
\text { artefakte }\end{array}$ & $\begin{array}{l}\text { Muskeltonus } \\
\text { auf nahe Null } \\
\text { abgefallen, } \\
\text { gelegentlich } \\
\text { Zuckungen, } \\
\text { Reflexe } \\
\text { erloschen }\end{array}$ \\
\hline EEG & $\begin{array}{l}\text { Alphawellen } \\
\text { im Wechsel mit } \\
\text { Betaaktivität }\end{array}$ & $\begin{array}{l}\text { Generelle Ver- } \\
\text { minderung der } \\
\text { Alphawellen, } \\
\text { Auftreten von } \\
\text { flachen } \\
\text { Thetawellen }\end{array}$ & $\begin{array}{l}\text { Spindeln, } \\
\text { K-Komplexe, } \\
\text { Betawellen und } \\
\text { Thetawellen }\end{array}$ & $\begin{array}{l}20-50 \% \\
\text { Deltawellen, die } \\
\text { in Abschnitten } \\
\text { von Thetawellen } \\
\text { eingefügt sind }\end{array}$ & $\begin{array}{l}\text { Deltawellen } \\
\text { mit hoher } \\
\text { Amplitude } \\
(>75 \mu V) \text {. } \\
\text { Anteil } \\
50-100 \%\end{array}$ & $\begin{array}{l}\text { Betawellen } \\
\text { mit einzelnen } \\
\text { Alpha- und } \\
\text { Thetawellen, } \\
\text { Sägezahn- } \\
\text { wellen }\end{array}$ \\
\hline
\end{tabular}

und Wärme bringt, während im Wachsein Frust, Hektik, Termindruck, Konflikte u. a. auf dem Menschen lasten.

Jegliche Störung des Nachtschlafes wird daher von den Menschen als etwas Unangenehmes, als ein Eingriff in ihre Intimsphäre bewertet. Das Erwachen während des Nachtschlafes wird subjektiv als unangenehm erlebt und ruft nega-

tiv-emotionale Zustände, d. h. Streß hervor: Personen, die nicht einschlafen oder nach dem nächtlichen Erwachen nicht wieder einschlafen können, beschreiben diesen Zustand als eine "psychische Qual«, als »Folter der Seele«, als etwas Unerträgliches. Jeder erfahrene Schlafmediziner kennt derartige Aussagen. Schlafstörungen erzeugen immer einen starken Leidensdruck.

Tabelle 3: Gegenüberstellung von Delta- und REM-Schlaf

\begin{tabular}{l|l|l}
\hline EEG & Deltaschlaf & REM-Schlaf \\
\hline EOG & Deltawellen & $\begin{array}{l}\text { flache Thetawellen ähnlich } \\
\text { denen des Einschlafstadiums }\end{array}$ \\
\hline EMG & keine Reaktion & schnelle Augenbewegungen \\
\hline $\begin{array}{l}\text { Wachstumshormon } \\
\text { Proteinsynthese }\end{array}$ & erhöht & keine elektrischen Muskelpotentiale \\
\hline \begin{tabular}{l} 
Traumerlebnis \\
\hline $\begin{array}{l}\text { Vegetative Funktionen } \\
\text { und Stoffwechsel }\end{array}$
\end{tabular} & $\begin{array}{l}\text { verminder REM-Schlaf ein } \\
\text { sparflamme« }\end{array}$ & sehr aktiv \\
\hline $\begin{array}{l}\text { Geschlechtshormone } \\
\text { Schlafwandeln }\end{array}$ & normal & stark ausgeprägt \\
\hline \begin{tabular}{l} 
Sprechen im Schlaf \\
\hline
\end{tabular} & stark & hohe Aktivität \\
\hline
\end{tabular}

Zum Spitzenfeld der Ursachen, welche den Schlaf stören, gehört der Lärm, der in den letzten Jahren für viele Menschen erheblich zugenommen hat. So ergab eine Befragung im Jahre 1978 in Westberlin, daß die Menschen die Belastung durch Lärm doppelt so hoch einschätzten wie die durch Luftverschmutzungen, also ein Verhältnis 2:1. Eine Wiederholungsstudie im Jahre 1990 belegt, daß die wahrgenommene Belastung durch Lärm gegenüber der Luftverschmutzung um das Sechsfache, also auf ein Verhältnis 6:1 angestiegen ist [17]. Mittlerweile fühlen sich über zwei Drittel der Bevölkerung durch Verkehrslärm belästigt.

\section{Schlaf und Lärm}

Zum Thema "Schlaf und Lärm " gibt es eine Vielzahl von Untersuchungen, die in den letzten zehn Jahren wiederholt zusammengefaßt wurden [18-24]. Die Arbeiten belegen, daß Lärm den Schlaf stören kann. Das Ausmaß der Störung ist u. a. abhängig von

- der Intensität (Pegel),

- dem Spektrum,

- der Expositionsdauer und -struktur, - dem Zeitpunkt der Einwirkung, 
- dem Informationsgehalt des Lärms sowie von vielen endogenen und exogenen Faktoren.

$\mathrm{Zu}$ den wichtigsten endogenen Faktoren zählen: Hörfähigkeit, Alter, Regulationsfähigkeit, Einstellung zum Lärm, psychische und physische Verfassung, Persönlichkeitsmerkmale, Geschlecht und Lärmempfindlichkeit.

$\mathrm{Zu}$ den wichtigsten exogenen Faktoren zählen: Tageszeit, Beleuchtung, Schlafraumtemperatur, Schlafumfeld, Medikamente sowie Genußmittel (Alkohol, Kaffee, Tee usw.).

Lärm ist nicht nur ein physikalischer Reiz, der den Menschen belastet, sondern ein individuelles Erleben. Lärm erzeugt Emotionen, denn er belästigt, stört die Gedanken, erschreckt, erzeugt Angst, Aggressivität und andere negative Verhaltensweisen. Lärm ist für den Menschen ein sozio-psycho-physiologischer Wirkungsfaktor. Aus diesem Grund muß in der Lärmwirkungsforschung nicht nur die momentane Lärmexposition, sondern auch die psycho-emotionale Einstellung des Menschen zum Lärm und die daraus resultierende Reaktion, die lange Zeit anhalten kann, berücksichtigt werden.

Weil der Mensch den Lärm als Erlebnis wahrnimmt, ist die Wirkung von Lärm keine lineare Reiz-Reaktions-Beziehung, sondern eine Funktion nach dem Regelkreisprinzip, in welches z. B. Gedächtnisinhalte, Motivationserregung, Alter und die Regulationsfähigkeit mit einbezogen sind.

Bereits Untersuchungen von Lukas und Kryter [25] ergaben, daß ältere Menschen bei Fluglärm eher erwachen als jüngere. Die gleichen Fluggeräusche führten bei $72,2 \%$ der 62 - bis 72 jährigen, bei $10,5 \%$ der 41 - bis 45 jährigen und nur bei $0,9 \%$ der 17 - bis 18 jährigen zum Erwachen.

Balzer et al. [26] untersuchten die Streßregulationsfähigkeit nach einem ähnlichen Schema, wie es Jansen et al. [27] in ihrer Arbeit beschreiben. Der Test dauerte 20 Minuten. Gemessen wurde die Regulation der elektrischen dermalen Aktivität (EDA). Zehn Minuten nach Beginn der Untersuchung wurde für die Dauer von einer Minute ein Licht-Klick-Reiz (eine Serie Stroboskopblitze und ein Lärmpegel von ca. $70 \mathrm{~dB}$ ) appliziert. Die Ergebnisse lassen deutlich vier unterschiedliche Streßregulationsmechanismen erkennen:

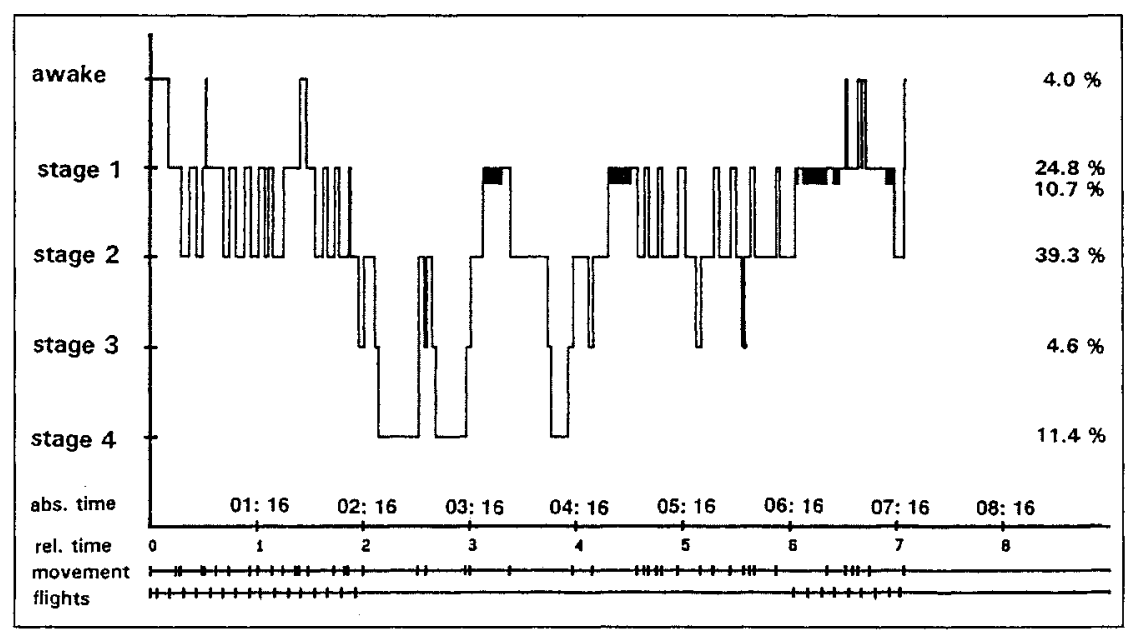

Abbildung 3: Typisches Schlafzyklogramm bei nächtlichem Fluglärm. Die Versuchsperson schlief bei 26 von 32 nächtlichen elektroakustisch simulierten Überflügen - letzteres entsprach einem Mittelungspegel von $L_{e q}$ innen $=53 \mathrm{~dB}(A)$ - im Schlaflabor des Instituts für Technische Akustik.

$37,4 \%$ der Untersuchten reagierten weder während der Reizdarbietung noch danach (Streßbeherrscher). 21,7\% antworteten heftig auf die Stimuli und fanden unmittelbar danach wieder ihre Regulationsstabilität (Streßbewältiger). $24,8 \%$ reagierten nicht auf die Reizdarbietung, sondern erst danach (Streßkompensierer), und 16,1\% reagierten während der Reizdarbietung und in der Phase danach (Streßdysregulierer).

Die beiden Untersuchung belegen exemplarisch die große individuelle Reaktionsbreite bei der Einwirkung akustischer Reize. Bei häufiger oder langanhaltender Exposition sind andere Reaktionen zu erwarten [28].

In Abbildung 3 ist ein typischer durch nächtlichen Fluglärm gestörter Schlafverlauf abgebildet.

Vergleichen wir das Schlafzyklogramm des in Ruhe Schlafenden (Abb. 2) mit dem Schlafzyklogramm der durch Fluglärm gestörten Person (Abb. 3), so ist sowohl eine Reduzierung der Tiefund REM-Schlafphasen zu verzeichnen als auch eine Zerstörung der Zeitarchitektur (Schlafzyklen).

Die durch Lärm hervorgerufenen Schlafstörungen lassen sich anhand ihrer zeitlichen Folge in Primär-, Sekundär- und Tertiärreaktionen unterscheiden.

\section{Zu den Primärreaktionen gehören:}

- kurzfristige Änderungen im EEG (Nullreaktionen),
- Verflachung der momentanen Schlaftiefe bis hin zu Aufwachreaktionen (Stadienwechsel),

- Veränderungen der Schlafstadienverteilung,

- Zunahme (Dauer) der Zeiten hoher Muskelanspannung (Movementtime),

- Verlängerungen der Latenzzeiten (insbesondere der Einschlaflatenz), - Verkürzung der Gesamtschlafzeit, aber auch vegetative Reaktionen wie - Änderungen der Atemfrequenz,

- Änderungen der peripheren Durchblutung

- Änderungen des Stoffwechsels und

- Änderungen der Hormonausschüttung.

Sekundärreaktionen sind reversible Beeinträchtigungen des Allgemeinzustandes nach dem Erwachen. $\mathrm{Zu}$ ihnen gehören die

- Beeinträchtigung der physischen Verfassung,

- Beeinträchtigung der psychischen Verfassung,

- Beeinträchtigung des Wohlbefindens,

- Beeinträchtigung der Leistung,

- Beeinträchtigung der Konzentration,

- Beeinträchtigung des Immunsystems.

Tertiärreaktionen sind irreversible gesundheitliche Beeinträchtigungen. Unter dem Begriff Gesundheit ist das physische und psychische Leistungsvermögen zu verstehen, das sowohl eine optimale Beziehung zwischen Mensch und Umwelt, zwischen Individuum und $\mathrm{Ge}$ - 
sellschaft, als auch körperliches, geistiges und soziales Wohlbefinden sichert [10].

\section{Nächtlicher Lärm und Gesundheit}

Nächtliche Lärmbeschränkungen werden national und international als notwendig erachtet, sofern für die Exponierten eine lärmbedingte Gesundheitsbeeinträchtigung (Tertiärreaktionen) zu erwarten ist. In diesem Zusammenhang kommt der Frage, welche der genannten Primär- und Sekundärreaktionen (Sofortreaktionen) bei langanhaltender $\mathrm{Ex}$ position zu einer Gesundheitsbeeinträchtigung führen, eine besondere präventivmedizinische Bedeutung zu.

Die gesundheitlichen Auswirkungen von Lärm haben eine Latenzzeit von schätzungsweise fünf bis zehn Jahren, in denen die Betroffenen ebenso einer Vielzahl von nichtakustischen gesundheitsgefährdenden Bedingungen ausgesetzt sind. Es ist daher experimentell außerordentlich schwierig, eine lärmbedingte Gesundheitsbeeinträchtigung zu isolieren. Aus diesem Grund ist in der Lärmwirkungsforschung noch immer die Frage umstritten, welche Sofortreaktion (bei langanhaltender Exposition) eine Gesundheitsbeeinträchtigung hervorruft. So behaupten Jansen, Linnemeier und Nitzsche [27], daß beim gegenwärtigen Stand der Erkenntnisse die Schallschutzmaßnahmen weiterhin auf die Aufwachreaktionen abgestellt werden müssen. Als Eckwert für die $\mathrm{Be}-$ urteilung ist eine Aufwachschwelle von $\mathrm{L}_{\max }=60 \mathrm{~dB}(\mathrm{~A})$ heranzuziehen, wobei sechs Lärmereignisse und weniger in einer Nacht oberhalb dieser Weckschwelle noch als zumutbar anzusehen sind. Hierbei werden weder die Erlebniskomponente, noch die Zerstörung der Zeitstruktur, noch die Schlafstadienumverteilung, noch die »Kosten « einer Kompensation berücksichtigt.

Von der wissenschaftlichen Erkenntnis, welche Sofortreaktion eine Gesundheitsgefährdung anzeigt, hängt aber der präventivmedizinische Handlungsbedarf $a b$, da die verschiedenen Sofortreaktionen bei unterschiedlichen Minimalpegeln beobachtet werden können.

Die in der Literatur beschriebenen Wirkungsschwellen (effect level) unterscheiden sich aber nicht nur deutlich voneinander - sofern verschiedene Sofortreaktionen herangezogen werden -, sondern für die gleichen Sofortreaktionen liegen erwartungsgemäß auch un- terschiedliche Ergebnisse vor. Die endogenen und exogenen Untersuchungsbedingungen (Moderatoren) sind in den meisten vorliegenden Untersuchungen ungenügend berücksichtigt oder nicht bekannt.

Zur Bewertung dieser Ergebnisse ist es notwendig, nicht nur die vielfältigen lärmbedingten Sofortreaktionen zu registrieren, sondern ein "tragfähiges" Wirkungsmodell zu erarbeiten. Dazu ist es hilfreich, die vorliegenden Untersuchungen anhand der Untersuchungsmethodik in drei Gruppen zu unterteilen (vgl. [24]). Aufgrund dieser Einteilung können die »Stärken « und »Schwächen« der vorliegenden Untersuchungen bei einer Modellbildung berücksichtigt werden.

\subsection{Tierexperimente (meist im Labor)}

Verschiedene Tiergruppen werden einer (meist) hohen Lärmbelastung ausgesetzt und sowohl die Sofortreaktionen als auch die (physiologischen) Auswirkungen beobachtet.

\section{Vorteil:}

Durch die extremen Lärmbelastungen können irreversible physiologische Beeinträchtigungen mit Krankheitswert in handhabbaren Zeitspannen methodisch kontrolliert erzeugt und mit den Sofortreaktionen verglichen werden.

Nachteil:

Die Übertragbarkeit der Ergebnisse auf reale Lärmbelastungen und auf den Menschen ist nicht gesichert.

Diese Untersuchungen dienen in erster Linie den Grundlagen einer Modellbildung.

\subsection{Experimentelle Studien (meist} im Labor, aber auch im Feld)

\section{a) Längsschnittstudien}

Es wird die gleiche Personengruppe unter veränderten Lärmbelastungen beobachtet.

Vorteil:

Die lärmbedingten Sofortreaktionen können »isoliert« beobachtet werden, sofern die Moderatoren über die Versuchsdauer als konstant angesehen werden können.

\section{b) Querschnittstudien}

Es werden unterschiedliche Personengruppen unter verschiedenen Lärmbelastungen beobachtet.
Vorteil:

Es können verschiedene Lärmbelastungen gleichzeitig untersucht werden, sofern die Gruppen hinsichtlich aller Moderatoren vergleichbar sind.

Nachteil der experimentellen Studien: Bei üblichen Versuchszeiten können keine Habituationsprozesse berücksichtigt werden. Es können keine direkten Aussagen über die gesundheitlichen Auswirkungen gewonnen werden.

Diese Untersuchungen sind in ibrer Gesamtheit geeignet, Wirkungsmodelle $a b-$ zusichern oder $z u$ modifizieren.

\subsection{Epidemiologische Studien (im Feld)}

\section{a) Kohortenstudien}

Personengruppen mit unterschiedlicher (natürlicher) Lärmexposition werden über einen längeren Zeitraum beobachtet. Die mittleren Unterschiede der erhobenen Sofortreaktionen und/oder des Gesundheitszustandes werden dem Lärm zugeordnet.

Vorteil:

Es können direkte Aussagen über Lärmwirkungen bei realen Belastungen gewonnen und mit den (habituierten) Sofortreaktionen verglichen werden.

\section{b) Fall-Kontrollstudien}

Es werden Personen, die bereits an bestimmten Krankheiten leiden (Fälle) hinsichtlich ihrer Lärmsituation unter Beachtung der Lebensweise mit solchen verglichen, die gesund sind (Kontrollen). Die mittleren Unterschiede in der Lärmsituation bei vergleichbarer Lebensweise werden als gesundheitsbeeinträchtigend angesehen.

Vorteil:

Risikoeinschätzungen über eine Gesundheitsbeeinträchtigung durch Lärm sind innerhalb kurzer Zeit möglich.

Nachteil der epidemiologischen Studien:

Es wirken neben dem Lärm eine unbekannte Zahl anderer gesundheitsrelevanter Einflußgrößen, die bei der Datenerhebung nicht alle kontrolliert werden können.

Diese Untersuchungen sind in ibrer Gesamtheit geeignet, Zusammenhänge zwischen Lärmbelastung und Gesundbeitsbeeinträchtigung abzuscbätzen.

Keine der beschriebenen Untersuchungsmethoden ist für sich allein ge- 
nommen in der Lage, die Kausalkette »nächtlicher Verkehrslärm - Sofortreaktion - Gesundheitsbeeinträchtigung " nachzuweisen.

Die Validität einer Sofortreaktion als Indikator für eine Gesundheitsbeeinträchtigung zeigt sich sowohl in der Reproduzierbarkeit bei Studien mit vergleichbarer Untersuchungsmethodik als auch in übereinstimmenden Ergebnissen von Studien mit unterschiedlichem Untersuchungsansatz.

- Die Ergebnisse von Tierexperimenten decken grundsätzlich Wirkungszusammenhänge auf und sind insbesondere bei geringer Kenntnis über den Forschungsgegenstand angemessen.

- Die Ergebnisse von experimentellen (Längsschnitt-)Studien am Menschen sind geeignet, um Wirkungsmodelle (unter realer Schallexposition) abzusichern und gegebenenfalls zu modifizieren.

- Aus den Ergebnissen von großangelegten epidemiologischen Studien können die gesundheitlichen Auswirkungen abgeschätzt werden.

Übereinstimmende Ergebnisse insbesondere zwischen experimentiellen und epidemiologischen Studien sind daher die wesentlichen Bausteine für ein Kausalmodell »nächtlicher Verkehrslärm Sofortreaktion - Gesundheitsbeeinträchtigung «.

Ergebnissen aus epidemiologischen Studien, die nicht durch ein Wirkungsmodell abgesichert werden können, ist ebenso mit Skepsis zu begegnen wie Wirkungsmodellen aus Tier-oder experimentiellen Studien, deren Auswirkungen nicht in epidemiologischen Studien erfaßt werden.

Unter diesem Gesichtspunkt kommt der Untersuchung der nächtlichen lärmbedingten Streßreaktion eine besondere Bedeutung zu. Sie liefert ein Wirkungsmodell, das die Ergebnisse experimentieller und epidemiologischer Untersuchungen vereint.

\section{Lärm und Streß}

Das biologische Streßkonzept nach Selye [29] beinhaltet die unspezifischen Reaktionsweisen des Organismus auf die Einwirkung unterschiedlicher exogener Reizeinflüsse, z. B. aus dem Lebensumfeld des Menschen. Dabei wird die unmittelbare physische Wirkung objektiver, physikalischer oder chemischer Reize betrachtet, nicht aber mög- liche psychische Vermittlungsprozesse. Somit wird beim Streß ein allgemeines, weitgehend stereotypes, reizunspezifisches Verhaltensmuster ausgelöst, das in erster Linie aus neuralen und humoralen Prozessen besteht und mit einer Steigerung des emotionalen Erregungsniveaus verbunden ist.

Nicht nur Extremsituationen wie Flucht, Kampf, Unfall und Schmerz führen zu Streßreaktionen, sondern auch alltägliche Situationen können zur Streßauslösung Anlaß geben. Neben den klassischen Streßsituationen, wie körperliche und geistige Anstrengung, Arbeiten unter Zeitdruck, Mißerfolg oder Bewegungseinschränkung, sind als Umweltstressoren Kälte- oder Hitzeeinwirkungen und die Lärmbelastung zu nennen. Streß ist heute zu einem zentralen Begriff in den verschiedensten wissenschaftlichen Disziplinen geworden, sowohl in der Biologie, der Medizin, der Psychologie und Soziologie, als auch in den Umwelt- und Ingenieurwissenschaften.

\section{Die Physiologie der Streß- reaktion}

Jede Streßreaktion beinhaltet den Versuch des Organismus, das durch die primäre »Alarmreaktion« gestörte physiologische Gleichgewicht durch adaptative Prozesse wiederherzustellen. Streß ist somit Störungsinduktion und Anpassungsreaktion zugleich. Zweck der Streßreaktion ist sowohl die schnelle Energiemobilisierung für evtl. erforderlich werdende plötzliche Anforderungen an den Muskelapparat als auch die Einstellung des Körpers auf zu erwartende extreme Funktionssteigerungen und Beanspruchungen bestimmter Organe oder Organsysteme, z. B. durch verstärkte Durchblutung, Stoffwechselaktivierung oder Stimulation der Abwehrmechanismen.

Je nach Auswirkungen und physiologischer Belastung durch die Streßreaktion muß zwischen Eustreß und Distreß unterschieden werden, wobei letzterer eine negative Beanspruchung, z. B. infolge akuter Reizeinwirkung mit mangelhafter oder fehlender Regulation oder infolge chronischer Reizüberflutung mit Dekompensation der regulativen Prozesse, darstellt. Dagegen handelt es sich beim Eustreß um eine positive Beanspruchung, die subjektiv z. B. als Erfolgserlebnis empfunden wird.

Eine wichtige Stellung bei der allgemeinen Steuerung der Streßreaktionen nimmt der Hypothalamus ein, wobei dieser Zwischenhirnabschnitt die Funktionen des somatischen, vegetativen, hormonellen und zentralen Aktivierungssystems koordiniert.

Die physiologische Streßreaktion ist an zwei übergreifende Systeme gebunden, das zentrale Nervensystem (ZNS) und das endokrine System.

\subsection{Das zentrale Nervensystem (neuronale Prozesse)}

Bei den neuronalen Prozessen müssen nochmals zwei Systeme unterschieden werden, die beide vom $Z$ wischenhirn gesteuert werden, jedoch unterschiedliche funktionelle Schwerpunkte haben: das vegetative Nervensystem (VNS) und das aufsteigende retikuläre Aktivierungssystem (ARAS).

a) Das vegetative Nervensystem (VNS)

Der Streßzustand beginnt mit einer »vegetativen Gesamtumschaltung «, wobei das VNS die Körperperipherie aktiviert und die Organfunktionen im Hinblick auf die Konstanthaltung des inneren $\mathrm{Mi}$ lieus sowie die Umstellung des Organismus auf Leistungsanforderungen reguliert und koordiniert. Man unterscheidet zwischen dem zentralen VNS, das hauptsächlich vom Hypothalamus gesteuert wird, und dem peripheren VNS, das in parasympathisches und sympathisches System aufgeteilt wird. Für eine Untersuchung der Streßreaktion ist vor allem letzteres von Bedeutung, da dieses für die Freisetzung der Katecholamine aus dem Nebennierenmark und der Synapsen sorgt.

\section{b) Das aufsteigende retikuläre Aktivie- rungssystem (ARAS)}

Das aufsteigende retikuläre Aktivierungssystem (ARAS) aktiviert die Hirnrinde und reguliert unter anderem den Wach-Schlaf-Rhythmus des Gehirns. Eine führende Rolle in diesem System spielt die Formatio reticularis im Subkortex, einem Neuronengeflecht, das vom Hirnstamm bis ins Zwischenhirn reicht. Sie wird einerseits durch Sinnesreize, andererseits auch durch das limbische System, das sog. »emotionale Gehirn«, angeregt. Somit können sowohl innere und äußere Reize als auch Erinnerungen und Gefühle über diese Formation den Schlaf bzw. das Schlaferleben beeinflussen.

\subsection{Das endokrine System (neurohu- morale Prozesse)}

Von zentraler Bedeutung sind hier die streßbedingten Hormonausschüttun- 
gen. Die Streßhormone werden in erster Linie auf dem Blutweg zu Organen oder Organsystemen transportiert. Die Steuerung der Hormonausschüttung erfolgt meist über negative Rückkopplung, das heißt, die Wirkung der Hormone wird an die produzierenden Stellen gemeldet, wo die Ausschüttung entsprechend gesteuert wird. Somit sind die Hormone nicht nur regelnde, sondern auch geregelte Größen. Sie werden, sofern ihre Wirksamkeit nicht bereits durch Stoffwechselprozesse (z. B. in dic Leber) oder durch Hormonantagonismen reduziert wurde, im Harn ausgeschieden.

Der Hypothalamus beeinflußt das endokrine System einerseits über die $\mathrm{Hy}$ pophyse (Freisetzung von Cortisol), andererseits auf neuralem Wege über den Sympathikus (Freisetzung von Katecholaminen). So sind zwei "Regel kreise zu beschreiben, die bei der Untersuchung von Streßreaktionen besondere Aufmerksamkeit verdienen.

a) Der Hypothalamus-HypophysenNebennierenrinden-Regelkreis

Die hormonelle Streßreaktion beginnt mit der Produktion von sog. Releasing factors im Hypothalamus, die zur Adenohypophyse (dem Hypophysenvorderlappen) transportiert werden, wo sie die Hormonbildungszellen stimulieren. Neben anderen wird hier das ACTH (adrenocorticotropes Hormon) gebildet und ausgeschüttet. Dieses Hormon wiederum löst eine Kette von Reaktionen aus, die eine Streßreaktion kennzeichnen, u. a. wird dabei die Funktion der Nebennierenrinde aktiviert. Die Nebennierenrinde produziert ihrerseits verstärkt Hormone, die Glucocorticoide, deren wichtigstes in diesem Zusammenhang das Cortisol ist. Diese Glucocorticoide wirken auf verschiedene Erfolgsorgane ein, zusätzlich beeinflussen sie Hypothalamus und Adenohypophyse im Sinne der beschriebenen negativen Rückkopplung.

\section{b) Der Hypothalamus-Nebennieren- mark-Regelkreis}

Das zentrale endokrine Organ dieses Regelkreises ist das Nebennierenmark. Dieses wird durch den Sympathikus aktiviert und produziert die Katecholamine Adrenalin und Noradrenalin, die meist sofort ins Blut freigesetzt werden. Die Katecholamine ermöglichen beispielsweise eine flexible Reaktion des Organismus auf emotionale Streßsituationen, wie Ärger, Angst oder Freude, und wirken insbesondere auf das HerzKreislauf-System, die Atmung, die Darmtätigkeit, den Stoffwechsel, das Pupillenspiel und das zentrale Nervensystem (ZNS) ein, wobei letzteres besonders durch Adrenalin beeinflußt wird.

\section{Operationalisierung der Lärmstreßreaktion}

Erreicht ein Geräusch das menschliche Ohr, wird es genau dann zum adäquaten Reiz, wenn das Spektrum des Geräusches nach Frequenz und Intensität hörbare Werte aufweist. Die durch adäquate Schallreize ausgelösten Nervenimpulse gelangen über die Hörnerven zur Hörrinde und zur Formatio reticularis. Lärm kann den Körper so zu einer "Alarmreaktion « veranlassen, bei der zunächst auf nervalem Weg die Katecholamine der Nebennierenrinde ausgeschürtet werden. Zeitlich verzögert wird ACTH ausgeschüttet, das seinerseits die Ausschüttung der Glucocorticoide und der Katecholamine stimuliert.

Glucocorticoide und Katecholamine wirken auf Erfolgsorgane ein und beeinflussen z. B. das Herz-Kreislaufsystem, den Stoffwechsel und die Blutfette. Jene werden zum großen Teil mit dem Harn ausgeschieden. Gleichzeitig wird über das aufsteigende retikuläre Aktivierungssystem der Schlafverlauf und sein subjektives Abbild, das Schlaferleben, beeinflußt.

Die primäre Operationalisierung einer Lärmstreßreaktion ist demzufolge über die Ausscbeidungsmengen der Katecholamine bzw. des Cortisols im Sammelurin nachweisbar, und die Stärke der Streßreaktion kann aus deren Konzentrationen abgelesen werden.

Die Änderung von vegetativen Reaktionen, z. B. der peripheren Durchblutung und des Stoffwechsels, stellen in diesem Modell Folgeerscheinungen der Streßreaktion dar und können als sekundäre Operationalisierung der Streßreaktion betrachtet werden.

Sowohl bei primärer wie sekundärer Operationalisierung ist zu beachten, daß die Streßreaktion eine reizunspezifische Reaktion darstellt. Die Methodik zur Untersuchung von Lärm-StreßReaktionen ist so auszulegen, daß der ursächliche Zusammenhang zwischen appliziertem Reiz und nachgewiesener Streßreaktion belegbar ist.

\section{Schlußfolgerungen}

Grundlage der Physiologie des Schlafes ist seine zeitliche Gliederung, d. h. seine Schlafstruktur. Als Schlafstruktur wird der rhythmische Ablauf des Schlafes bezeichnet, der durch das Schlafpolygramm verifiziert wird. Hierzu zählen der Wechsel von NONREM- und REM-Schlaf sowie die Schlafzyklen und die Abfolge der Schlafstadien. Wird diese Schlafstruktur durch Lärm gestört, dann formiert sich der unterbrochene Schlaf von neuem (z. B. [30, 31]). Wir erhalten bei häufigen Störungen einen fragmentierten, d. h. zerhackten Schlafverlauf. Das bedeutet, daß der Schlafende immer wieder gezwungen wird, einen neuen Schlafzyklus zu beginnen, wozu ein hoher Energieaufwand im Sinne der Streßtheorie notwendig ist.

Untersuchungen von Dement [32-34] belegen, daß massive Störungen des Schlafes innerhalb von wenigen Tagen zu einer nachhaltigen Zerstörung der Schlafstruktur und damit zu einer Zerstörung der individuellen biologischen Rhythmen führen. Gleichzeitig sind bei den Betroffenen Erhöhungen der Streßhormone (Kortisol) im Blut, Gedächtnis- und Konzentrationsstörungen, Schwindelgefühle, Kopfschmerzen usw. zu verzeichnen $[4,11]$. Die schlafgestörten Personen benötigten nach Beendigung der Störung mehrere Tage bis zur völligen Normalisierung ihrer Schlafstruktur. Ein fragmentierter Schlafverlauf kennzeichnet eine starke negative Beanspruchung infolge exogener oder endogener Reizeinwirkung.

Diese Form der Schlafstörung und ihre langfristigen Auswirkungen wurden nicht nur im Zusammenhang mit Lärm, sondern auch weltweit im Zusammenhang mit der Schlafapnoe (Atemstillstandshypoxie) untersucht. Hier liegen Untersuchungen an mehreren hunderttausend Personen vor (u. a. [35-41]). Nach übereinstimmenden Erkenntnissen kann die beschriebene Schlafstörung und ihre Auswirkungen in fünf Stufen eingeteilt werden:

- Es treten Mikroarousel im SchlafEEG auf, die als unbewußtes Erwachen (Subererwachen) interpretiert werden können.

- Der Schläfer verläßt sein augenblicklich ablaufendes Schlafstadium.

- Der Schlafzyklus wird unterbrochen, so daß er wieder neu begonnen werden muß. 
- Bei häufiger Störung der Schlafzyklen kommt es zur pathologischen Schlafstadienfragmentierung; die Schlafeffizienz wird erheblich gesenkt.

- Langfristige Schlafstadienfragmentierung führt zur Zerstörung der individuellen Zeitstruktur; die Gesundheit ist gefährdet.

Die permanente Störung des Schlafes ist als starker Distreß zu interpretieren, der das interne Milieu nachweislich verändert. Aus diesem Grund ist aus präventivmedizinischer Sicht ein ungestörter und ausreichender Schlaf von großer Bedeutung für die Gesundheit. Langfristige Schlafstörungen stellen eine Gesundheitsgefährdung dar.

\section{Literatur:}

[1] Peter, J. H., Köhler, D., Knob, B., Mayer, G., Penzel, T., Raschke, F., und Zulley, J. (Hrsg) Weißbuch Schlafmedizin. Regensburg: Roderer Verlag 1995.

[2] Billiard, M.: Die Zukunft der Schlafmedizin in Europa. In: K. Meier-Ewert, E. Rüther (Hrsg.): Schlafmedizin. Jena: Gustav Fischer Verlag 1993.

[3] Schramm, E., und Riemann, D.: JCSD - Internationale Klassifikation der Schlafstörungen, Deutsche Ausgabe. Weinheim: Beltz Verlags Union 1993.

[4] Moore-Ede, M.: Die Nonstop-Gesellschaft. München: Heyne Verlag 1993.

[5] Cross National Medication Survey, 1990. Zitiert in [2].

[6] Dement, W.C., Carskadon, M.A., and Ley, R.: The prevalence of narcolepsy. Sleep Res. 147, 2 (1973).

[7] Billiard, M., et al. 1991. Zitiert in [2]

[8] Ekbom, K.A.: Restless legs syndrome. Neurol. 10 (1960) 868-873.

[9] Bixler, E. O., Kales, A., Soldatos, C. R., Kales, J. D., and Healey, S.: Prevalence of Sleep Disorders in Los Angeles Metropolitan Area. Am. J. Psychiat. 136 (1979) 1257-1262.

[10] Hecht, K.: Schlaf und die GesundheirsKrankheitsbeziehung unter dem Aspekt des Regulationsbegriffes von Virchow. In Hecht, K., Engfer, A., Peter, H. J., und Poppei, M. (Hrsg): Schlaf, Gesundheit, Leistungsfähigkeit. Berlin/Heidelberg: Springer-Verlag 1993

[11] Koella, P. W.: Die Physiologie des Schlafes. Stuttgart/New York: Gustav Fischer Verlag 1988.

[12] Hecht, K.: Besser schlafen, schöner träumen. Südwestverlag 1993
[13] Maschke, C.: Der Einfluß von Nachtfluglärm auf den Schlafverlauf und die Katecholaminausscheidung. Dissertation TUBerlin 1992.

[14] Maschke, C., Arndt, D., Ising, H., Laude, G., Thierfelder, W., und Contzens, S.: Der Einfluß von Nachtfluglärm auf die Streßhormonausscheidung von Flughafenanwohnern. Abschlußsbericht DFG $\mathrm{Nr}$. Gr 452/8-2. Stuttgart/New York: Gustav Fischer Verlag 1995.

[15] Winson, J.: Neurobiologie des Träumens. Spektrum der Wissenschaft, Januar 1991

[16] Horne, J. A.: Why we sleep - the function of sleep in humans and other mammals. New York/Tokio: Oxford University Press 1988.

[17] Kruppa, B., Babisch, W., und Ising, H.: Die Hauptstörfaktoren im Wohnungsumfeld einer repräsentativen Berliner Bevölkerungsstichprobe und die Rolle des Lärms. Forum Städte und Hygiene 1994.

[18] Eberhardt, J. L., and Akselson, K. R.: The disturbance by road traffic noise of sleep. J. of Sound and Vibration 114, 3 (1987).

[19] Eberhardt, J.L.: The influence of road traffic noise on sleep. J. of Sound and Vibration $1127(3),(1988)$

[20] Gottlob, D.: Störungen des Nachtschlafs durch Fluglärm. Vortrag vor der Fluglärmkommission Frankfurt, 1989.

[21] Griefahn, B.: Präventivmedizinische Vor schläge für den nächtlichen Schallschutz, Zeitschrift für Lärmbekämpfung 37 (1990).

[22] Linnemeier, A. F. B.: Die Auswirkungen von Lärm auf den natürlichen Nachtschlaf des Menschen - Versuch einer Klassifikation und Bewertung von Veröffentlichungen von 1984 bis 1993. Dissertation, Düsseldorf, eingereicht 1995.

[23] Maschke, C., Druba, M., und Pleines, F.: Beeinträchtigung des Schlafes durch Lärm. Umweltbundesamt 1996 (noch unveröffentl.).

[24] Paschier-Vermeer, W.: Noise and health. The Hague: Health Council of the Netherlands publication no. 360-31 (1995).

[25] Lukas, J.S., and Kryter, K.D.: Awakening effects of simulated sonic booms and subsonic aircraft noise. In: Welch/Welch (Eds.) Physiological effects of noise. New York: Plenum Press 1970.

[26] Balzer, H. W., and Hecht, K.: Categorisation of different personality types with respect to susceptibility to stress and coping skill, Eighth international Montreaux Congress on stress. Montreaux, Switzerland 1996.

27] Jansen, G., Linnemeier, A., und Nitzsche M.: Methodenkritische Überlegungen und Empfehlungen zur Bewertung von Nachtfluglärm. Zeitschr. für Lärmbekämpfung 42 , 4 (1995).

[28] Graff, Ch., Bockmühl, F., und Tietz, V. Lärmbelastung und arterielle Hypertoniekrankheit beim Menschen. In: Nitschkoff, St.,
Kriwizkaja, G. (Hrsg): Lärmbelastung, akustischer Reiz und neurovegetative Störungen. Leipzig: Verlag Georg Thieme 1968.

[29] Selye, H.: The stress of life. McGraw-Hill Verlag 1956

[30] Richter, H. R.: Schlafstörungen durch Verkehrslärm. Zeitschr. für Lärmbekämpfung 1969.

[31] Richter, H. R.: Elektroencephalographische Befunde bei Schlafstörungen durch Verkehrslärm und ihre Bedeutung für die prophylaktische Medizin resp. die Verkehrsplanung. In: Broda, E., Locker, A., Springer-Lederer, A. (Hrsg.): First European Biophysics Congress. Verlag der Wiener Medizin Akademie 1971.

[32] Dement, W.C.: The effect of dream deprivation. Science 131 (1960)

[33] Dement, W. C., Greenberg, S., and Klein, R. The effect of partial REM sleep deprivation and delayed recovery. J. Psychiatric Res. 1966.

[34] Dement, W. C.: The biological rule of REM sleep. In: Kales, A. (ed.): Sleep: Physilogy and Pathology 1969.

[35] Dorow, P., und Thalhofer, S.: Verlaufsbeobachtungen von Patienten mit schlafbezogener Atemregulierungsstörung. In: Hecht, K., Engfer, A., Poppei, M.: Schlaf, Gesundheit, Leistungsfähigkeit. Springer-Verlag 1993.

[36] Fietze, I., Warmuth, R., Quispe-Bzavo, S. und Reglin, B.: Erste Erfahrungen bei der Diagnose und Therapie von schlafbezogenen Atmungsstörungen im Schlaflabor der Charité. In: Hecht, K., Engfer, A., Poppei, M. Schlaf, Gesundheit, Leistungsfähigkeit. Springer-Verlag 1993.

[37] Guilleminault, C., and Quera-Selve, M. A. Obstructive sleep apnoea: is prevention eve possible? Eur. Respir. J. Suppl. 1990.

[38] Lavie, P., Halperin, E., Zomer, J., and Alroy, G.: Across-night lengthening of sleep apneic episodes. Sleep 1981.

[39] Peter, H.J., Podszus, T., Becker, H., und von Wichert, P.: Schlafbezogene Atmungsstörungen - Schlafapnoe. Dt. Ärztebl. 1989

[40] Peter, H. J., Faust, M., Fett, I., Podszus, T., Schneider, H., Weber, K., und von Wicherr, P.: Die Schlafapnoe. DMW 1990.

[41] Peter, H.J.: Schlafbezogene Atmungsstörungen - eine Herausforderung für die pathologische Physiologie. In: Hecht, K., Engfer, A., Poppei, M.: Schlaf, Gesundheit, Leistungsfähigkeit. Springer Verlag 1990.

Anschrift der Verfasser:

Dr. C. Maschke, TU Berlin, Institut für Technische Akustik, Einsteinufer 25, 10587 Berlin, Dr. H. Ising, Institut für Wasser-, Boden- und Lufthygiene des Umweltbundesamtes, Corrensplatz 1, 14195 Berlin, und Prof. Dr. med. K. Hecht, Institut für Streßforschung, Chausseestr. 111, 10115 Berlin 